\title{
3D MECHANICAL CHARACTERIZATION OF ARTIFICIAL MUSCLES WITH STEREOSCOPIC COMPUTER VISION AND ACTIVE CONTOURS
}

\author{
R. Verdú, R. Berenguer *, J. Morales, G. Vázquez, T.F. Otero, L.Weruaga \\ Cartagena University of Technology, Spain. E-mail: rafael.verdu@upct.es \\ * Catholic University of Murcia, Spain. E-mail: rberenguer@pdi.ucam.edu
}

\begin{abstract}
Artificial muscles are formed by attaching a conducting polymeric film to a non-conducting one. Applying an electrical current on the muscle, a macroscopic bending movement appears on it. Study of curvature variations and related parameters, such as speed of motion or energy of curvature, is necessary for improving the efficiency of these devices. In a previous work, a one-cam computer vision system was developed to estimate motion parameters in 2D with precise results. In this paper, a two-cam stereo vision system is proposed to process the image sequence and track the muscle in 3D. Active contours models are employed in motion detection and mechanical parameters estimation. Results prove the validity of this approach, allowing automatic testing on the research into artificial muscles.
\end{abstract}

\section{INTRODUCTION}

Some polymer-based devices are able to convert electrochemical energy into mechanical energy producing a macroscopic mechanical work. which results into a bending movement along the polymeric stripe. Based on this concept, multilayer polymeric devices, known as artificial muscles, have been constructed and patented [1], [2].

Studies to characterize the mechanical properties have been conducted using a single camera system and active contours (snakes). This approach provides the relevant parameters for the muscle modelling [3]. Since only one camera is used, a unique condition is required for the observation. namely the position of the single camera is forced to be perpendicular to the muscle motion. Therefore, more accurate and camera-independent results could be achieved by using a dual camera system.

The aim of this paper is to propose an automatic method to characterize the mechanical properties of the artificial muscles in 3D. This method is based on a stereoscopic vision system that avoids the limitations of the $2 \mathrm{D}$ analysis.

From this analysis, relevant conclusions about the behavior of the device can be drawn. These studies are essen-

This work was supported by MCYT BQU2001-0477 tial, in particular in order to determine the feasibility of their commercial exploitation in a later stage.

This paper is structured as follows: Section 2 contains the system and method proposed in this paper for characterizing the mechanical properties of the described polymeric device; Section 3 shows some experimental results of this system, which prove the validity of the approach; and finally, Section 4 summarizes the conclusions and the shows the further work.

\section{MECHANICAL CHARACTERIZATION WITH A STEREO-VISION SYSTEM}

The proposed system architecture is composed of two fully controllable Pan/Tilt/Zoom cams, connected to a personal computer remotely via RS-232C VISCA ${ }^{T M}$ interface.

An application is specially developed to perform all the process. Firstly, it controls the cams motion in order to track the target. It grabs a stereo-video with the moving polymeric film. In addition, from each stereo-frame and cams parameters, we estimate the requested artificial muscle 3D parameters. The processing phases performed are described in the following subsections.

\subsection{System calibration and target identification}

A particular scheme for a stereo vision system is used. Cams are placed orthogonally each other in the $\mathrm{XY}$ plane in front of the moving muscle. Assuming that both cams work with the same tilt angle $\alpha$ and the distance $d$ between each cam and target is equal, the $\mathrm{XZ}$ and $\mathrm{YZ}$ projection of the poly. meric film can be easily obtained.

A system calibration at the set up process of the application is necessary for improving the quality of the parameter estimation. After the calibration process, the system identifies the target maximizing its view in both cams. For this purpose the system adjusts cam parameters using the tilt and zoom movements at both cams simultaneously. 


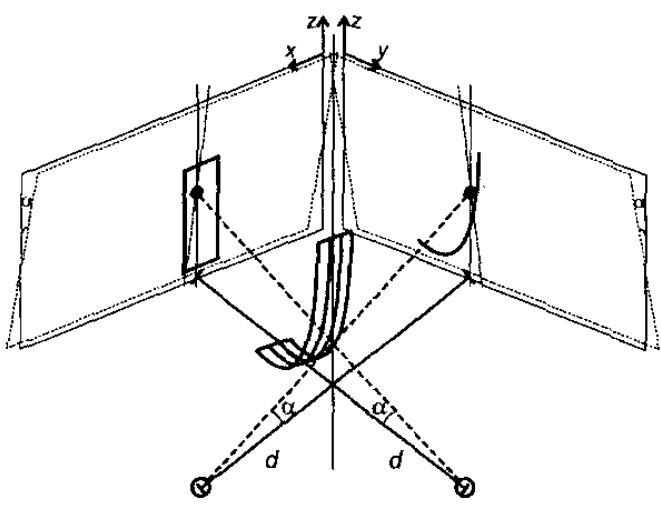

Fig. 1. System scheme.

\subsection{Video grabbing process and preprocessing}

A multichannel grab card is used to acquire the sequence of stereo frames. The position and zoom of the cameras are fixed during the grabbing process. These parameters are stored within the video for the processing phase.

Over each acquired frame and before proceeding to estimate the motion between them, simple automatic preprocessing tasks are performed: In order to obtain the projection over the $\mathrm{XZ}$ and $\mathrm{YZ}$ plane, a linear projection with angle $\alpha$ is applied (see figure 1); the polymeric film image is segmented by applying a threshold; the resulting shape is refined applying morphological operations, which leads to a thin $2 \mathrm{D}$ curve that defines the $\mathrm{XZ}$ or $\mathrm{YZ}$ projection of inner film contour.
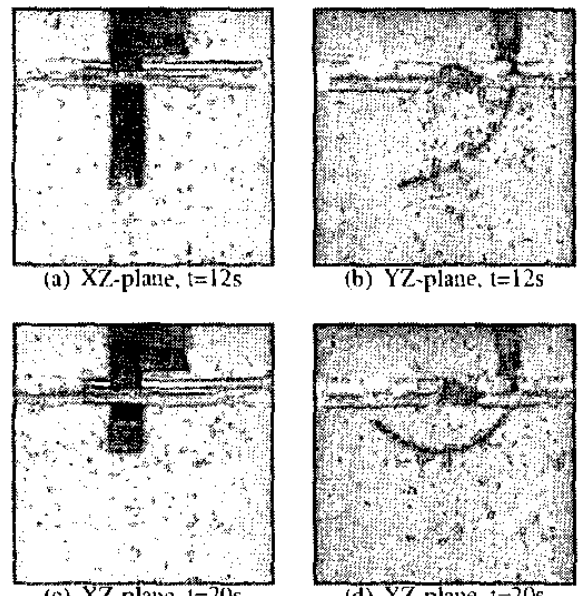

(c) XZ-plane, $1=20 \mathrm{~s}$

(d) YZ-plane, $t=20 \mathrm{~s}$

Fig. 2. XZ and $Y Z$ projection of the muscle movement.

\subsection{Frame-to-frame 2D contour estimation by snakes}

The polymeric stripe experiences a bending movement that, although not complex, is clearly non-rigid. Despite the popularity of block-matching methods for estimating the spatial correspondence between pairs of images, the task of tracking non-rigid motion is better accomplished with deformable models and snakes [4]. Snakes are used successfully in the 2D approach [3].

In the 3D approach, the tracking of the same object is simultaneosly accomplished from multiple camera views. By tracking this object in different projections independently, shape inconsistencies can not be prevented, as it is shown in figure 2 .

In [5] and [6] an unique 3D snake is utilized in the 3D curve estimation. An altemative approach for this problem is introduced instead. We would have two time-varying parametric contours:

$$
\begin{aligned}
& v_{x z}(s, t)=(x(s, t), z(s, t))^{T} \\
& v_{y z}(s, t)=(y(s, t), z(s, t))^{T}
\end{aligned}
$$

$v_{x z}(s, t)$ in the left image plane $(x, z) \in \mathbb{R}^{2}$ and $v_{y z}(s, t)$ in the right image plane $(y, z) \in \mathbb{R}^{2}$ where $x, y$ and $z$ are functions of the length index $s \in[0, L]$ and time $t$.

The shape of the contour into each image $I_{x z}(x, z)$ and $I_{y z}(y, z)$ is dictated by the following energy functional:

$$
\begin{aligned}
& \mathcal{E}\left(v_{x z}\right)=\mathcal{S}\left(v_{x z}\right)+\mathcal{P}\left(v_{x z}\right) \\
& \mathcal{E}\left(v_{y z}\right)=\mathcal{S}\left(v_{y z}\right)+\mathcal{P}\left(v_{y z}\right)
\end{aligned}
$$

respectively. Where $S(v)$ is the internal energy controlled by the parameters "tension" and "rigidity" of the snake and $\mathcal{P}(v)$ refers to the external energy, calculated through the gradient of each image [4].

$$
\begin{gathered}
\mathcal{S}(v)=\frac{1}{2} \int_{0}^{L} \alpha(s)\left|\frac{\partial v}{\partial s}\right|^{2}+\beta(s)\left|\frac{\partial^{2} v}{\partial s^{2}}\right|^{2} d s, \\
\mathcal{P}(v)=\int_{0}^{L} \mathcal{P}_{I}(v) d s,
\end{gathered}
$$

Both snakes are in equilibrium when equations (1) and (2) reach their minimum independly. This state can be accomplished with the Lagrange algorithm on a dynamic snake [7]. Additional adjustments are made in the algorithm to take into account the specific mechanical aspects of the problem: Given that the film bends and does neither shrink nor enlarge, the snake length is forced to stay constant at any time; this feature is implemented by using an additional internal force applied into each node; and finally, the first node of the snake is initially attached to the upper extreme of the stripe and kept fixed throughout the whole motion estimation process. 


\subsection{D contour construction}

Once we have the two $2 \mathrm{D}$ contours, the construction of the $3 \mathrm{D}$ contour can be achieved by assuming that the $z$ coordinate is the same for both curves and the two camera views are orthogonal. For this first approach, $v(s, t)=$ $(x(s, t), y(s, t), z(s, t))$ can be obtained taking $x$ from $v_{x z}$ and $y$ from $v_{y z}$. This approach is appropriate with nonoverlapping muscle movements.

Since the algorithm tracks the muscle motion -attaching the snake to the muscle projection- a problem rises when the muscle bending movement reaches 90 degrees, as it is shown in the left side of figure 2 . In this case, the lower extreme of the snake would be attached to the lower extreme of the projection, instead of the lower extreme of the muscle.

In order to solve this occlusion problem and enhance the accuracy in the curve estimation, a second approach to the problem is proposed. After each iteration of the 2D algorithm, the $\mathrm{z}$ value for each node of the $2 \mathrm{D}$ snake is pondered from both $2 \mathrm{D}$ curves. Different algorithms have been tested to detect and prioritize the non-overlapping projection of the $z$-coordinate above the other one.

\subsection{Parametric motion and mechanical properties}

From the 3D contour, we can obtain the key parameters for describing the mechanical properties of the muscle.

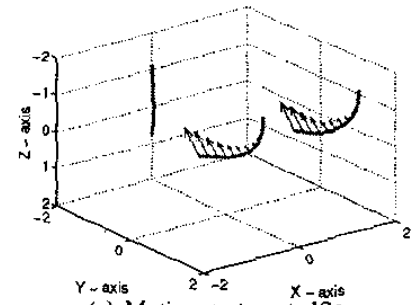

(a) Motion vectors, $\begin{aligned} & X \text {-axis } \\ & t=12 s\end{aligned}$

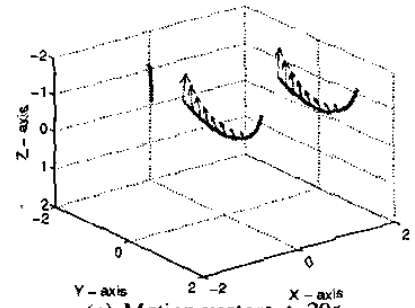

(c) Motion vectors, $\begin{aligned} & \mathrm{x}=20 \mathrm{ax} \\ & \mathrm{y}\end{aligned}$

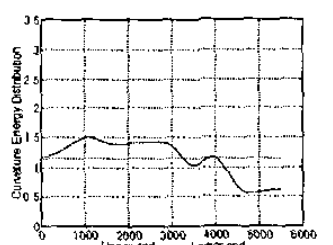

(b) Energy, $t=12 \mathrm{~s}$

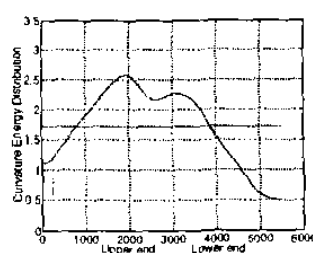

(d) Energy, $t=20 \mathrm{~s}$
Fig. 3. Motion and curvature energy distribution.

\section{Parametric motion}

Considering a constant muscle length during the movement and a properly adjusted snake to the muscle in every frame, $v(s, t)=(x(s, t), y(s, t), z(s, t))$, the resulting displacement is given by the difference between each snake position in two correlative stereo-frames. The velocity can be obtained as the rate of the positional changes by multiplying the displacement vectors by the frame rate:

$$
\begin{aligned}
\overrightarrow{v e l}(s, t) & =(\Delta x(s, t), \Delta y(s, t), \Delta z(s, t)) / \Delta t \\
\Delta x(s, t) & =x(s, t+\Delta t)-x(s, t) \\
\Delta y(s, t) & =y(s, t+\Delta t)-y(s, t) \\
\Delta z(s, t) & =z(s, t+\Delta t)-z(s, t)
\end{aligned}
$$

where $\Delta t$ is the sampling period between two consecutive frames, $\Delta t=1 / f p s$ and $f p s$ is the frame rate.

\section{Energy of curvature}

The curvature of a contour at a point can be defined as the inverse of the radius of the arc that overlays the contour at that point [8]. Since the curvature is a function of radius, a scale-invariant version of the curvature defined by the following equation:

$$
E_{c u r v}^{e}(s)=\frac{\left|\hat{n} \cdot v_{s s}\right|}{\left|v_{s}\right| \pi}
$$

where $\hat{n}$ is a unitary vector normal to the contour, $v_{s}=$ $\left(x_{s}, y_{s}, z_{s}\right), v_{s s}=\left(x_{s s}, y_{s s}, z_{s s}\right)$ and $x_{s}, x_{s s}, y_{s}, y_{s s}, z_{s}$ and $z_{s s}$ are the first and second derivatives of $x, y$ and $z$ components respect to the parameter $s$. Integrating over each element in the contour and summing the resulting integrals, results into the total energy for the entire contour.

\section{EXPERIMENTAL RESULTS}

In the experiment, the movement of the free end of the film was caused by the flow of a $+3 \mathrm{~mA}$ constant current.

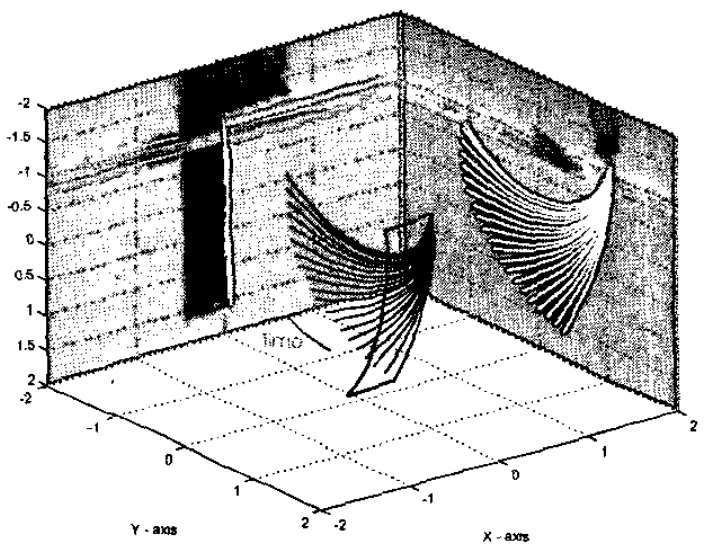

Fig. 4. Motion of the muscle during the experiment.

In figure 2, stereo-frames of the acquired video sequence are presented, which show the artificial muscle at different 

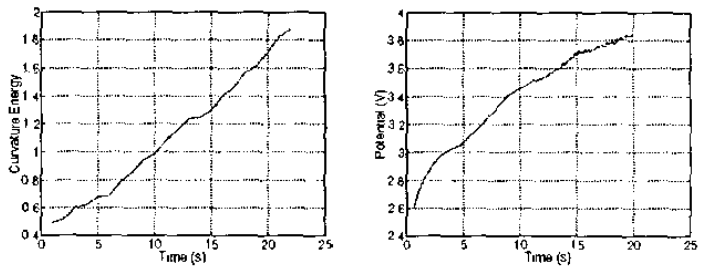

Fig. 5. Curvature energy and tension versus time.

times. In the left side of figure 3 the motion occurred in the frames of the previous figure is shown: This motion throughout the film length in terms of direction and magnitude is represented by displacement vectors. The right side of same figure shows the resulting distribution of the curvature along the muscle for the corresponding frames. In figure 4 , the set of contours that describe the trajectory of the film during the experiment is shown. Each pair of consecutive contours are equally time-spaced. In the left hand side of figure 5 the whole curvature of the muscle versus time is shown, and the right hand side of this figure shows the measured voltage in the muscle upper end, generated by the $+3 \mathrm{~mA}$ constant current intensity.

As in the 2D approach [3], it can be observed that there is a linear relationship between the muscle curvature energy and the device consumed load. It proves the high efficiency of the device, so that most of the supplied energy is employed to make the muscle bend.

From figure 2, it can be observed, that the muscle movements occurs only in the plane parallel to the muscles layers. Unlike in the 2D approach, with the stereo system proposed, it is not necessary that the direction of the muscle movement takes place perpendicular to the camera focal axis. This enables the study of imperfections in muscles movements or the research about more complex multilayer devices, with 3D movements.

\section{CONCLUSIONS AND FURTHER WORK}

In this paper, an improved computer vision-based method to quantify the mechanical properties of polymeric artificial muscles has been described. This method is based on active contours algorithms for 3D tracking of non-rigid moving objects. The main result of this new approach is the ability to derive the parameters related to the motion, such as speed, magnitude and curvature distribution of the polymeric film in any direction of the space. Note that this key result cannot be achieved in a single camera system. These values are compared with the electrochemical magnitudes resulting during the experimental process, such as consumed energy, voltage and current applied. The study performed on a wet multilayer polymeric device reveals interesting results: The energy consumed is directly related to the curvature suffered by the muscle; the distribution of the degree of bending along the muscle contour keeps nearly constant during the whole motion cycle -from the equilibrium to a high bending state-; this distribution could be related to quality and efficiency of the manufacturing process.

Finally, the current work lines consist of an improved automatic calibration and target identification system, in order to perform automatic testing of artificial muscles. Synchronization between the muscles power supply and testing system is required for this purpose too. In addition, the width of the polymer strip will be considered in future works. This new feature will require to model the muscle on an active surface instead of on an active contour to study the torsion movement.

\section{REFERENCES}

[1] T. F. Otero. Patent ep-9200095 and ep-9202628, 1992.

[2] T. F. Otero and J. M. Sansiena. "Bilayer dimensions and movement of artificial muscles". Bioelectrochem. Bioenergetics, 47, 1997.

[3] R. Verdú, J. Morales, A. J. Fernandez-Romero, M. T. Cortés, T. F. Otero and L. Weruaga. "Mechanical characterization of artificial muscles with computer vision". 9th Annual International Symposium on Smart Structures and Materials. Proceedings of the SPIE, 2002.

[4] D. Terzopoulos, M. Kass and A. Witkin. "Snakes: active contour models". International Journal of Computer Vision, pages 321-331, 1998.

[5] C. Sbert and A. F. Sole, "Stereo reconstruction of 3D curves". International Conference on Pattern Recognition (ICPR'O0), 2000.

[6] P. Radeva, R. Toledo, C. Von Land and J, Villanueva. "3D vessel reconstruction from biplane angiograms using snakes". Computers in Cardiology, 25:773-776, 1998.

[7] D. Terzopoulos, J. Liang and T. McInerney. "United snakes". Proceedings of the 7th IEEE International Conference on Computer Vision, pages 933-940, 1999.

[8] K. A. Stroud. Engineering Mathematics. McMillan. 